\title{
Bolgiano scale in confined Rayleigh-Taylor turbulence
}

\author{
G. Boffetta ${ }^{1} \dagger$, F. De Lillo ${ }^{1}$, A. Mazzino ${ }^{2}$ and S. Musacchio ${ }^{3}$ \\ ${ }^{1}$ Dipartimento di Fisica Generale and INFN, Università di Torino, via P. Giuria 1, 10125 Torino, Italy \\ 2 Dipartimento di Fisica, Università di Genova, INFN and CNISM, via Dodecaneso 33, \\ 16146 Genova, Italy \\ 3 CNRS, Lab. J.A. Dieudonné UMR 6621, Parc Valrose, 06108 Nice, France \\ (Received 21 July 2011; revised 12 September 2011; accepted 6 October 2011; \\ first published online 25 November 2011)
}

\begin{abstract}
We investigate the statistical properties of Rayleigh-Taylor turbulence in a threedimensional convective cell of high aspect ratio, in which one transverse side is much smaller that the others. By means of high-resolution numerical simulation we study the development of the turbulent mixing layer and the scaling properties of the velocity and temperature fields. We show that the system undergoes a transition from a three- to two-dimensional turbulent regime when the width of the turbulent mixing layer becomes larger than the scale of confinement. In the late stage of the evolution the convective flow is characterized by the coexistence of Kolmogorov-Obukhov and Bolgiano-Obukhov scaling at small and large scales, respectively. These regimes are separated by the Bolgiano scale, which is determined by the scale of confinement of the flow. Our results show that the emergence of the Bolgiano-Obukhov scaling in Rayleigh-Taylor turbulence is connected to the onset of an upscale energy transfer induced by the geometrical constraint of the flow.
\end{abstract}

Key words: turbulence simulation, turbulent convection

\section{Introduction}

Turbulent thermal convection appears in many natural phenomena, from heat transport in stars to turbulent mixing in the atmosphere and the oceans, as well as in technological applications (Siggia 1994; Ahlers, Grossmann \& Lohse 2009). Turbulent convection is driven by buoyancy forces generated by temperature differences. These are then mixed by the turbulent flow itself up to small scales at which molecular diffusivity becomes important. A fundamental problem in turbulent convection is the determination of the statistical properties of velocity and temperature fluctuations in the inertial range of scales in which turbulent mixing is at work.

A first step in this direction was taken by Obukhov (1949) and Corrsin (1951) who generalized the Kolmogorov argument for the statistics of a temperature field in the so-called passive limit, in which the effects of the buoyancy forces on the velocity field are neglected. In this limit the temperature and velocity fluctuations at scales within the inertial range are both characterized by a Kolmogorov scaling exponent 1/3. 
This picture is, a posteriori, consistent with the assumption that the buoyancy force becomes less and less important at small scales where therefore the passive limit is recovered (Shraiman \& Siggia 1990).

An alternative prediction was proposed by Bolgiano (1959) and Obukhov (1959) in discussing the statistics of velocity and temperature fluctuations in a stably stratified atmosphere. The buoyancy forces allow the introduction of a characteristic scale, the Bolgiano scale $L_{B}$, above which buoyancy becomes important and the statistics of the velocity and temperature are determined by the balance between buoyancy and inertia forces. This leads to a different prediction for the scaling exponents, namely $3 / 5$ for the velocity and $1 / 5$ for the temperature fields. In spite of many years of experimental and numerical investigations, no clear evidence of this scenario has been given (see the recent review by Lohse \& Xia 2010).

In this paper we study turbulent convection within the simplified Rayleigh-Taylor framework. In particular we focus on the case in which the convective flow is confined in a box with one of the dimensions (e.g. y) much smaller than the other two. In the absence of convection, the effects of the aspect ratio on the dynamics of fluid layers has been widely investigated both numerically (Smith, Chasnov \& Waleffe 1996; Ngan, Straub \& Bartello 2005; Celani, Musacchio \& Vincenzi 2010) and experimentally (Shats, Byrne \& Xia 2010; Xia et al. 2011). The results reported in these works show that confinement induces a reduction of dimensionality of the flow at large scales, from three- to two-dimensional behaviour. Specifically, in Smith et al. (1996) and Celani et al. (2010) it has been observed that small-scale three-dimensional turbulence, characterized by a forward cascade of kinetic energy, can coexist with a two-dimensional inverse cascade, which takes place at scales larger than the scale of confinement.

Here we investigate, by means of state-of-the-art numerical simulations of primitive equations, the consequences of the confinement on convective turbulence in the case of the Rayleigh-Taylor configuration. We show that a dimensional transition occurs in the flow when the width of the mixing layer becomes larger than the confining scale $L_{y}$, which becomes the Bolgiano scale of the system. After the transition we find the appearance of coexistent Kolmogorov-Obukhov scaling at scales smaller than $L_{y}$ and Bolgiano scaling at scales larger than $L_{y}$. We conjecture that the geometrical interpretation of the Bolgiano scale as the confining scale could be more general than the present setup and therefore we suggest a new direction for numerical and experimental investigations of scaling properties in thermal convection.

The rest of this paper is organized as follows. In $\S 2$ we introduce the equations of motion and we provide the theoretical background. In $\S \S 3$ and 4 we present and discuss the results from the numerical simulations while $\S 5$ is devoted to conclusions.

\section{Theoretical background and method}

Rayleigh-Taylor (RT) turbulence is one of the simplest configurations of thermal convection in which a cold, heavier layer of fluid is placed on top of a hot, lighter layer in a gravitational field. RT instability occurs in many phenomena ranging from geophysics to astrophysics and to technological applications (Isobe et al. 2005; Cabot \& Cook 2006; Schultz et al. 2006). The gravitational instability develops in an intermediate layer of turbulent fluid (the mixing layer) the width of which grows in time.

In this paper, we consider miscible RT turbulence at low Atwood numbers. Within the Boussinesq approximation, the equations for the dynamics of the velocity field 
$\boldsymbol{u}=(u, v, w)$ coupled to the temperature field $T(\boldsymbol{x}, t)$ (which is proportional to the density $\rho$ via the thermal expansion coefficient $\beta$ as $\rho=\rho_{0}\left[1-\beta\left(T-T_{0}\right)\right]$ where $\rho_{0}$ and $T_{0}$ are reference values) are:

$$
\begin{gathered}
\partial_{t} \boldsymbol{u}+\boldsymbol{u} \cdot \nabla \boldsymbol{u}=-\left(1 / \rho_{0}\right) \nabla p+v \nabla^{2} \boldsymbol{u}-\beta \boldsymbol{g} T, \\
\partial_{t} T+\boldsymbol{u} \cdot \nabla T=\kappa \nabla^{2} T,
\end{gathered}
$$

together with the incompressibility condition $\boldsymbol{\nabla} \cdot \boldsymbol{u}=0$. In (2.1) $\boldsymbol{g}=(0,0,-g)$ is the acceleration due to gravity, $v$ is the kinematic viscosity and $\kappa$ is the thermal diffusivity. The initial condition for the RT problem is an unstable temperature jump $T(\boldsymbol{x}, 0)=-\left(\theta_{0} / 2\right) \operatorname{sgn}(z)$ in a fluid at rest $\boldsymbol{u}(\boldsymbol{x}, 0)=0$. The temperature jump defines the Atwood number as $A=(1 / 2) \beta g \theta_{0}$.

As the system evolves, the available potential energy $P=-\beta g\langle z T\rangle$ is converted into kinetic energy $E=(1 / 2)\left\langle|\boldsymbol{u}|^{2}\right\rangle$ at a rate given by the energy balance:

$$
-\frac{\mathrm{d} P}{\mathrm{~d} t}=\beta g\langle w T\rangle=\frac{\mathrm{d} E}{\mathrm{~d} t}+\varepsilon,
$$

where $w$ is the vertical velocity and $\varepsilon=v\left\langle\left(\partial_{\alpha} u_{\beta}\right)^{2}\right\rangle$ is the viscous energy dissipation rate and angle brackets indicate volume average. From the dimensional balance between the loss of potential energy and the increase of kinetic energy one has that typical velocity fluctuations grow as $u_{r m s} \simeq \beta g \theta_{0} t$, and therefore the width of the turbulent mixing layer $h(t)$ grows following the accelerated law $h(t) \simeq \beta g \theta_{0} t^{2}$. The integral scale $L(t)$ of the turbulent flow, defined as the largest scale on which kinetic energy is injected, is expected to grow proportionally to the geometrical scale $h(t)$ as shown by Vladimirova \& Chertkov (2009) and Boffetta et al. (2010b).

According to the phenomenological theory of RT turbulence, developed by Chertkov (2003), the scaling behaviour of the velocity and temperature fluctuations in the range of scales between the integral scale $L$ and the dissipative scale $\eta$ strongly depends on the dimensionality of the flow. We report in the following the main points of the theoretical predictions.

For the three-dimensional (3D) case, one assumes that the buoyancy force $\beta \boldsymbol{g} T$ balances the inertia term in (2.1) at the integral scale $L(t)$ and becomes negligible as the cascade proceeds towards small scales, consistently with the Kolmogorov-Obukhov phenomenology. For velocity and temperature fluctuations $\delta u(r)=u(x+r)-u(x)(u$ denoting one velocity component) and $\delta T(r)=T(x+r)-T(x)$ one therefore expects

$$
\begin{aligned}
& \delta u(r) \simeq \varepsilon(t)^{1 / 3} r^{1 / 3}, \\
& \delta T(r) \simeq \varepsilon_{T}(t)^{1 / 2} \varepsilon^{-1 / 6} r^{1 / 3},
\end{aligned}
$$

where the energy dissipation rate $\varepsilon$ grows in time as $\varepsilon(t) \simeq\left(\beta g \theta_{0}\right)^{2} t$, adiabatically following the dynamics of the large eddies, while the temperature dissipation rate decreases as $\varepsilon_{T}(t) \simeq \theta_{0}^{2} t^{-1}$. The spatial and temporal scaling (2.3) have been numerically verified by Cabot \& Cook (2006), Boffetta et al. (2009) and Matsumoto (2009), who have also revealed the presence of small deviations due to intermittency.

The above scenario is not consistent in two dimensions (2D) where kinetic energy is transferred toward large scales developing an inverse cascade (as is usual in twodimensions, see e.g. Kraichnan \& Montgomery (1980)). In this case one has that the buoyancy term injects energy at all scales and therefore this is not a cascade in a strict sense as the energy flux is not constant in wavenumber. Velocity and temperature 
fluctuations follow in this case the Bolgiano-Obukhov scaling

$$
\begin{aligned}
& \delta u(r) \simeq \varepsilon_{T}(t)^{1 / 5}(\beta g)^{2 / 5} r^{3 / 5}, \\
& \delta T(r) \simeq \varepsilon_{T}(t)^{2 / 5}(\beta g)^{-1 / 5} r^{1 / 5}
\end{aligned}
$$

which has been verified in numerical simulations of 2D RT turbulence by Celani, Mazzino \& Vozella (2006) and Biferale et al. (2010).

Let us now consider a convective cell with large aspect ratio $L_{x}, L_{z} \gg L_{y}$. At short times, when $L(t)<L_{y}$ the turbulent flow in the mixing layer can be considered threedimensional and a direct cascade with Kolmogorov-Obukhov scaling (2.3) is expected. In this regime the Bolgiano scale, defined in terms of dissipations as (Lohse \& Xia 2010)

$$
L_{B}=(\beta g)^{-3 / 2} \varepsilon^{5 / 4} \varepsilon_{T}^{-3 / 4},
$$

grows in time proportionally to the width of the mixing layer and the integral scale, $L_{B}(t) \simeq \beta g \theta_{0} t^{2} \simeq L(t) \simeq h(t)$.

At later times, when $L(t)>L_{y}$ the flow cannot be fully three-dimensional. As a consequence of the geometrical constraint in the $y$-direction, a two-dimensional phenomenology might appear at scales $L_{y}<r<L(t)$. For this to happen, most of the energy injected by buoyancy forces on those scales should go to larger scales, producing an inverse cascade with Bolgiano-Obukhov scaling (2.4). However, a residual direct cascade to scales $r<L_{y}$ should be present with a small-scale flux $\varepsilon(t)$ given by the matching of the scaling (2.3) and (2.4) at $r=L_{y}$ :

$$
\varepsilon(t) \simeq\left(\beta g \theta_{0}\right)^{6 / 5} L_{y}^{4 / 5} t^{-3 / 5} .
$$

In this regime, the Bolgiano scale becomes time independent: using (2.6) and $\varepsilon_{T}(t) \simeq \theta_{0}^{2} t^{-1}$ in the definition (2.5) one obtains $L_{B}(t) \simeq L_{y}$. Therefore the transition from $3 \mathrm{D}$ to $2 \mathrm{D}$ turbulence fixes the value of the dynamical scale $L_{B}$ to the geometrical scale $L_{y}$ by reducing the energy flux to small scales.

The time associated with this transition is given by the continuity requirement in energy dissipation, i.e. equating (2.6) with 3D dissipation $\left(\beta g \theta_{0}\right)^{2} t$ which gives $\tau=\left(L_{y} /\left(\beta g \theta_{0}\right)\right)^{1 / 2}$.

Summarizing, the phenomenology that we expect for the long-time behaviour of RT turbulence with large aspect ratio is that shown in figure 2. At small scales $\eta \leqslant r \leqslant L_{y} \simeq L_{B}$ a three-dimensional direct cascade with Kolmogorov-Obukhov scaling and residual flux (2.6) is expected. At large scales $L_{y} \leqslant r \leqslant L(t)$ a two-dimensional inverse cascade with Bolgiano-Obukhov scaling should be observed.

The range of scales in which the Bolgiano-Obukhov phenomenology is expected becomes wider as the system evolves because the mixing layer width grows in time as $L(t) \sim t^{2}$. Conversely, the Kolmogorov-Obukhov scaling range shrinks, because the Kolmogorov scale grows as $\eta(t) \sim\left(v / \varepsilon_{v}(t)\right) \sim t^{3 / 20}$, and it should eventually vanish, provided that the system could evolve for arbitrarily long times.

\section{Numerical results for single-point statistics}

The predictions discussed in the previous section have been tested against the results of state-of-the-art high-resolution numerical simulations of (2.1), for large-aspect-ratio geometry with $L_{x}=2 \pi, L_{z} / L_{x}=2, L_{y} / L_{x}=1 / 32$, in which the resulting flow is thus strongly confined in the $y$-direction. The integration of (2.1), discretized on a $4096 \times 128 \times 8192$ grid with periodic boundary conditions, has been performed 


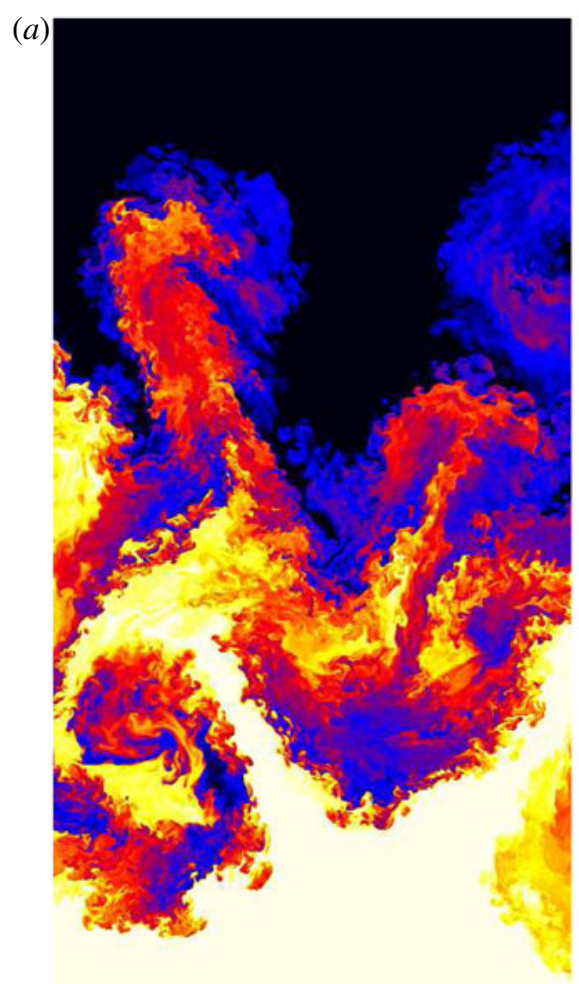

(b)

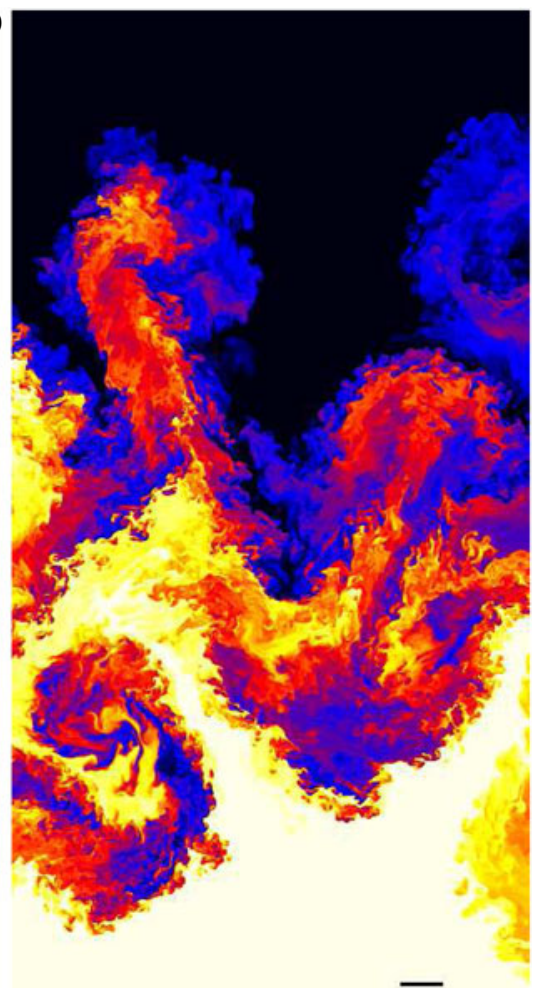

FIGURE 1. (Colour online available at journals.cambridge.org/flm) Central part of two vertical $(x, z)$ sections of the temperature field (white $=$ hot, black $=$ cold) from direct numerical simulations of Boussinesq equations (2.1) at resolution $4096 \times 128 \times 8192$ taken at time $t=30 \tau$ (see text for the definition of $\tau$ ): $(a)$ is taken at $y=0,(b)$ at $y=L_{y} / 2$. The black segment in $(b)$ represents the transverse lenght $L_{y}$. Parameters are $\beta g=0.5, v=\kappa=2 \times 10^{-5}$ and the initial temperature jump is $\theta_{0}=1$. The picture clearly shows that large-scale $y$-independent 2D structures coexist with small-scale 3D turbulence.

with a fully parallel pseudospectral code, with 2/3-dealiasing, running on a IBM-SP6 supercomputer.

Figure 1 shows two vertical sections of the temperature field taken at two different $y$ positions in the late stage of the simulations, when the amplitude of the mixing layer is much larger than $L_{y}$. We observe that small-scale 3D features are different in the two sections, while large-scale structures are clearly $y$-independent, indicating the two-dimensional nature of the large-scale flow. In spite of the complexity of the temperature field, the mean vertical temperature, obtained by averaging over horizontal plane $\bar{T}(z, t) \equiv\langle T(\boldsymbol{x}, t)\rangle_{x, y}$, has a simple monotonic profile which is found to evolve in self-similar way in time $\bar{T}(z, t)=\phi(z / h(t))$, where $h(t)$ defines the width of the mixing layer. The functional shape $\phi$ of the profile has been extensively studied in the 3D case where it is found to be well described by a cubic polynomial (Boffetta, De Lillo $\&$ Musacchio 2010a).

Figure 3 shows two temperature profiles from the present simulation. The first is computed at short time, when $h(t)<L_{y}$ and the flow is fully three-dimensional, 


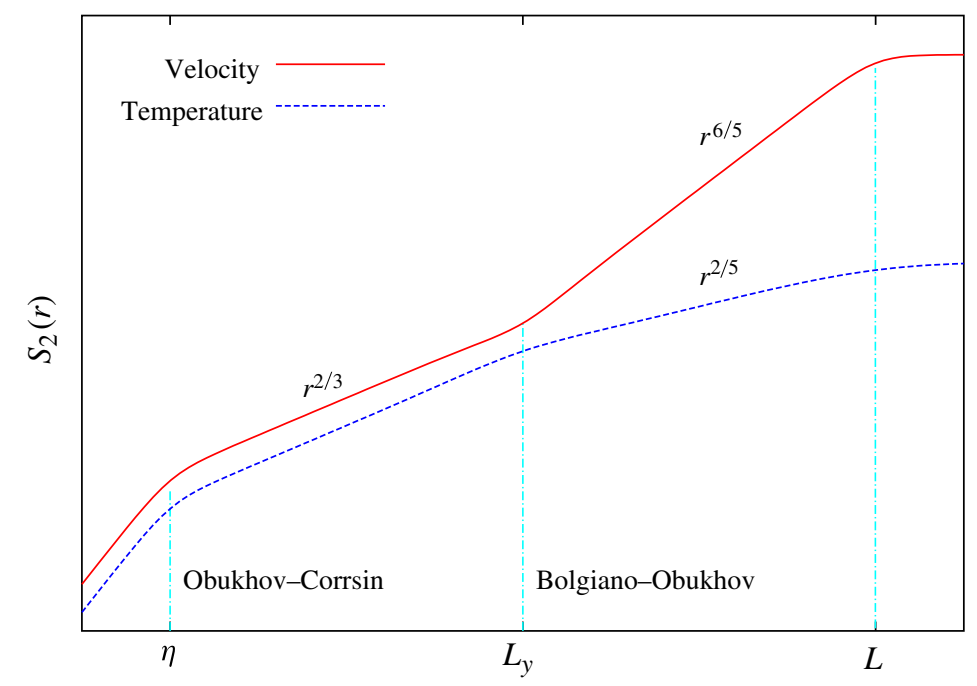

FIGURE 2. (Colour online) The scaling regimes for velocity structure function in quasi-2D Rayleigh-Taylor turbulence. $\eta$ represents the smallest Kolmogorov scale, $L$ is the integral scale.

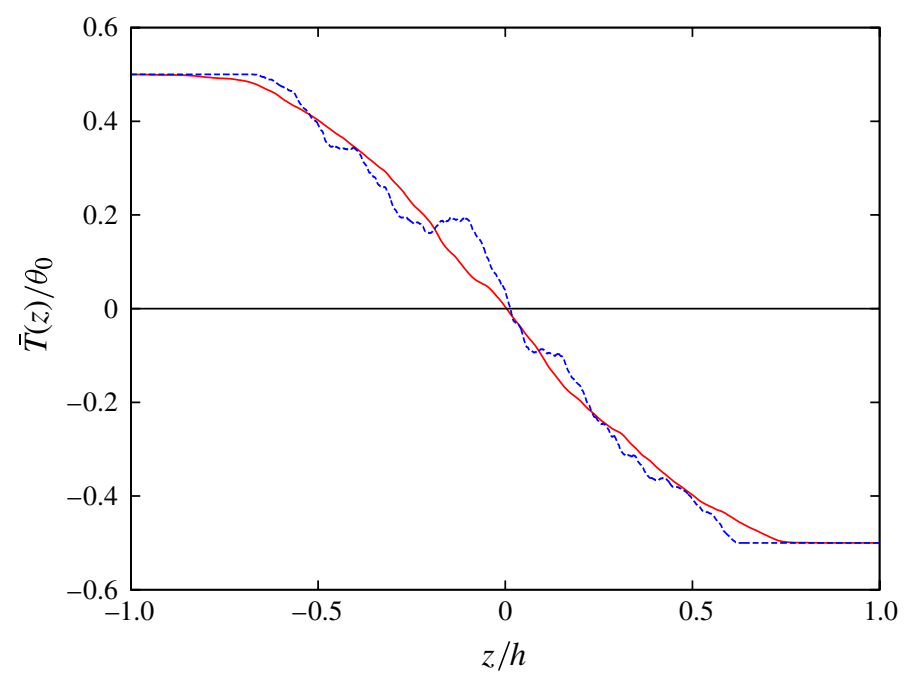

FIGURE 3. (Colour online) Mean vertical temperature profile $\bar{T}(z, t)$ at times $t=10 \tau$ (continuous line) and $t=30 \tau$ (dotted line).

while the second is obtained at late time (corresponding to figure 1) when $h(t)>L_{y}$. Fluctuations observed in the late profile are due to the presence of two-dimensional structures which are not averaged out on the $y$-direction, as shown in figure 1 .

By computing the mean profile at different times we are able to obtain the evolution of the width of the mixing layer $h(t)$. Several definitions of $h(t)$ have been proposed (Dalziel, Linden \& Youngs 1999; Cabot \& Cook 2006). Here we use the simple one based on a threshold, so that $h$ is defined by $\bar{T}( \pm h / 2)=\mp s \theta_{0} / 2$ (with $s=0.8$ ). As explained above, both in $2 \mathrm{D}$ and $3 \mathrm{D}$ we expect a quadratic law $h(t)=\alpha A g t^{2}$. The 


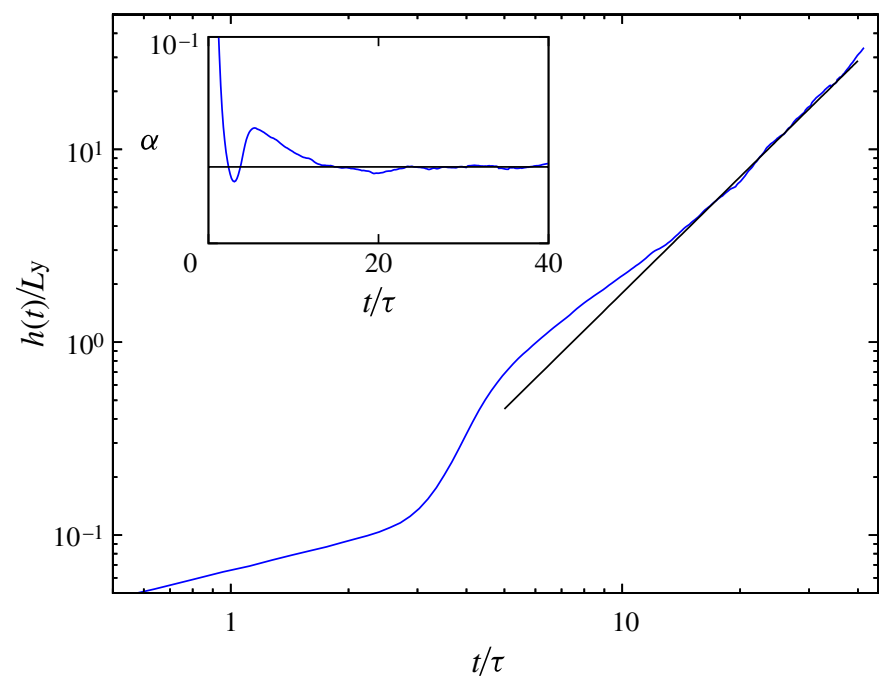

FIgURE 4. (Colour online) Temporal evolution of the mixing layer width $h(t)$. The straight line represents the quadratic law $h(t)=\alpha A g t^{2}$ with $\alpha=0.035$ obtained from the compensated plot of $h(t) /\left(A g t^{2}\right)$ shown in the inset.

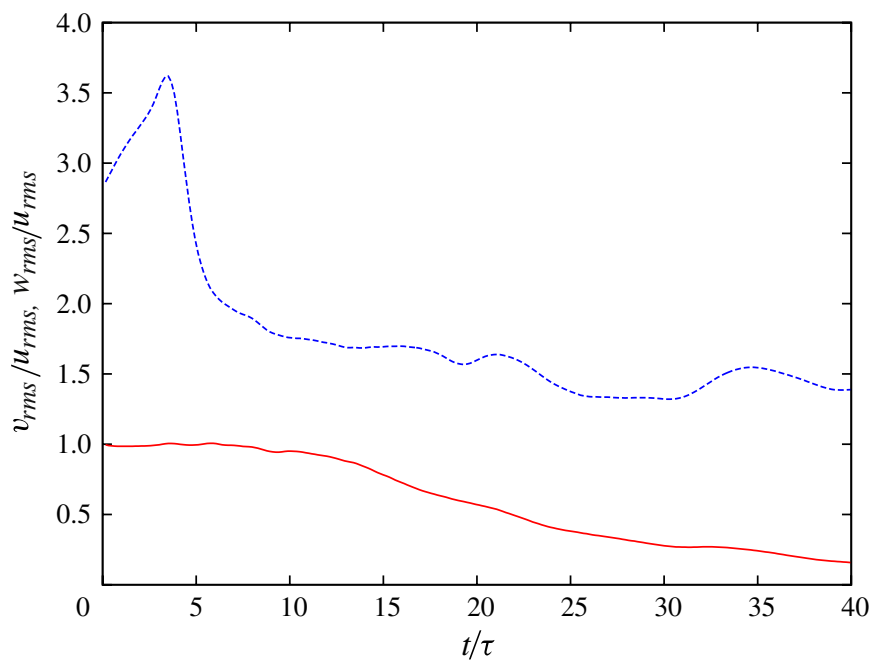

FIgURE 5. (Colour online) Temporal evolution of the ratio of $y$ component to $x$ component (continuous line) and of $z$ component to $x$ component (dashed line) of r.m.s. velocity fluctuations.

value of the dimensionless coefficient $\alpha$ has been measured by many authors for the 3D case (Ristorcelli \& Clark 2004; Cabot \& Cook 2006; Boffetta et al. 2010b). As shown in figure 4 , we observe at late times $(t>10 \tau)$ a quadratic growth for $h(t)$. This happens when $h(t)>L_{y}$ which therefore corresponds to a quasi-2D flow. The measured value of $\alpha$, simply obtained by compensating $h(t)$ with $A g t^{2}$ (inset of figure 4), gives $\alpha \simeq 0.037$, which is remarkably close to what has been obtained in 3D (Cabot 2006; Boffetta et al. 2010b). 


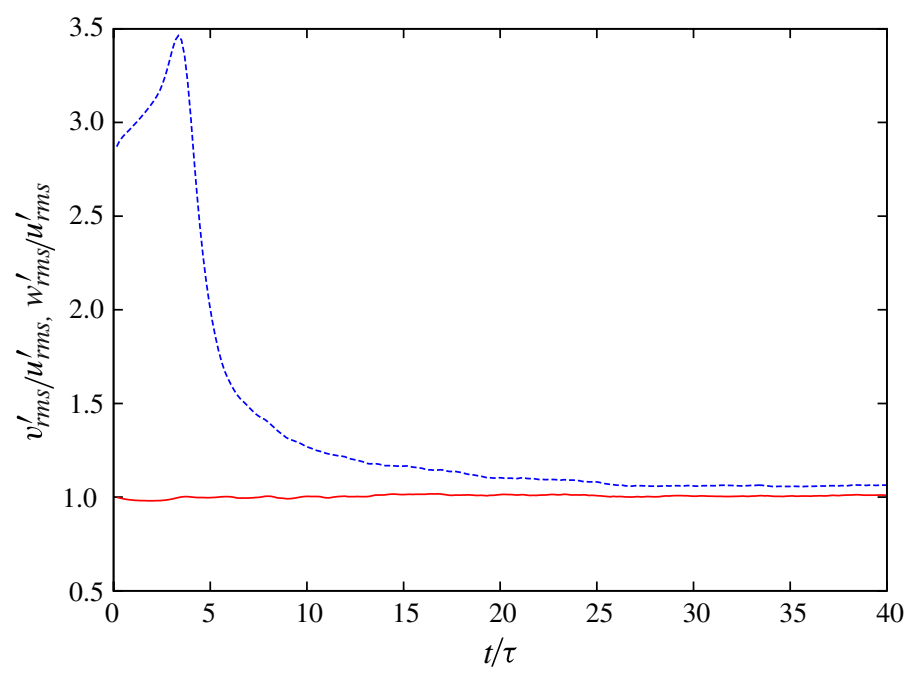

FIgURE 6. (Colour online) Temporal evolution of the ratio of $y$ component to $x$ component (continuous line) and of $z$ component to $x$ component (dashed line) of r.m.s. velocity gradients.

The first indication of bidimensionalization of the flow is given by the ratio of root-mean-square (r.m.s.) velocities. Figure 5 shows that for $t \geqslant 10 \tau$ the flow becomes increasingly anisotropic with $v_{r m s} \ll u_{r m s}$. The vertical velocity $w_{r m s}$, which at short time dominates because of the presence of vertical plumes, at late time is of the order of $u_{r m s}$ and their ratio remains constant. The isotropy of the flow is recovered at small scales, as is shown by the r.m.s. of velocity gradients (see figure 6). The r.m.s. gradients of the horizontal velocities $u_{r m s}^{\prime}=\sqrt{\left\langle\left(\partial_{\alpha} u\right)^{2}\right\rangle}$ and $v_{r m s}^{\prime}=\sqrt{\left\langle\left(\partial_{\alpha} v\right)^{2}\right\rangle}$ remain equal throughout the simulation. At late time also the r.m.s. gradient of vertical velocity $w_{r m s}^{\prime}=\sqrt{\left\langle\left(\partial_{\alpha} w\right)^{2}\right\rangle}$ becomes almost equal to that of the horizontal velocities, indicating the recovery of small-scale isotropy.

The transition from 3D to 2D turbulent behaviour is clearly signalled by a change in the ratio between the energy growth rate $\mathrm{d} E / \mathrm{d} t$ and the viscous dissipation rate $\varepsilon$ (the energy flux to small scales in the direct cascade) shown in figure 7 . In the 3D regime both these quantities grow linearly in time and therefore their ratio is constant. After the transition, the inverse cascade sets in and $(\mathrm{d} E / \mathrm{d} t) / \varepsilon \sim t^{8 / 5}$, as follows from both (2.6) and $E \sim u_{r m s}^{2} \sim t^{2}$.

Figure 8 shows the evolution of the Bolgiano scale (2.5) as a function of time. In the first stage, $L_{B}$ grows proportionally to the width of the mixing layer (see inset). After the transition it saturates to a value close to $L_{y}$ as a consequence of the reduction of $\varepsilon$.

\section{Two-point statistics}

In the late stage of the evolution, when $L(t)>L_{y}$ we expect a turbulent mixing layer with the simultaneous presence of a direct and an inverse cascade in the two ranges of scales $r<L_{y}$ and $r>L_{y}$, respectively. Figure 9 shows the kinetic energy spectra of the 2D components of velocity $E_{u}(k)+E_{w}(k)$ and of the transverse component $E_{v}(k)$ as a function of 2D wavenumber $k=\sqrt{k_{x}^{2}+k_{z}^{2}}$ and $k_{y}=0$. At large scales, fluctuations of transverse velocity are suppressed and the spectrum of the $2 \mathrm{D}$ velocity field is compatible with Bolgiano scaling $k^{-11 / 5}$, in agreement with the theoretical 


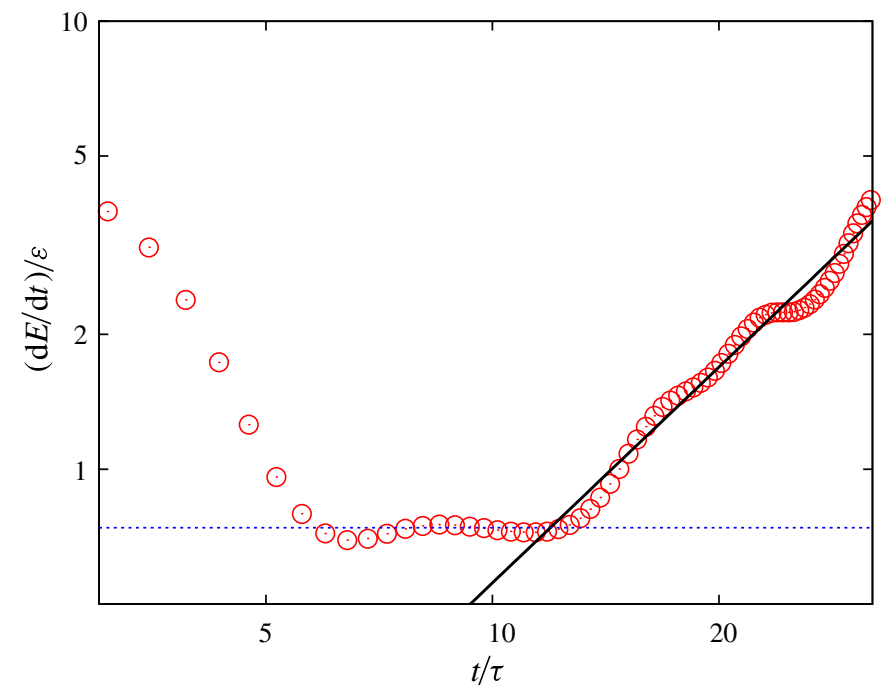

FIGURE 7. (Colour online) The ratio of the kinetic energy growth rate to viscous energy dissipation rate as a function of time. The solid straight line represents the behaviour $t^{8 / 5}$.

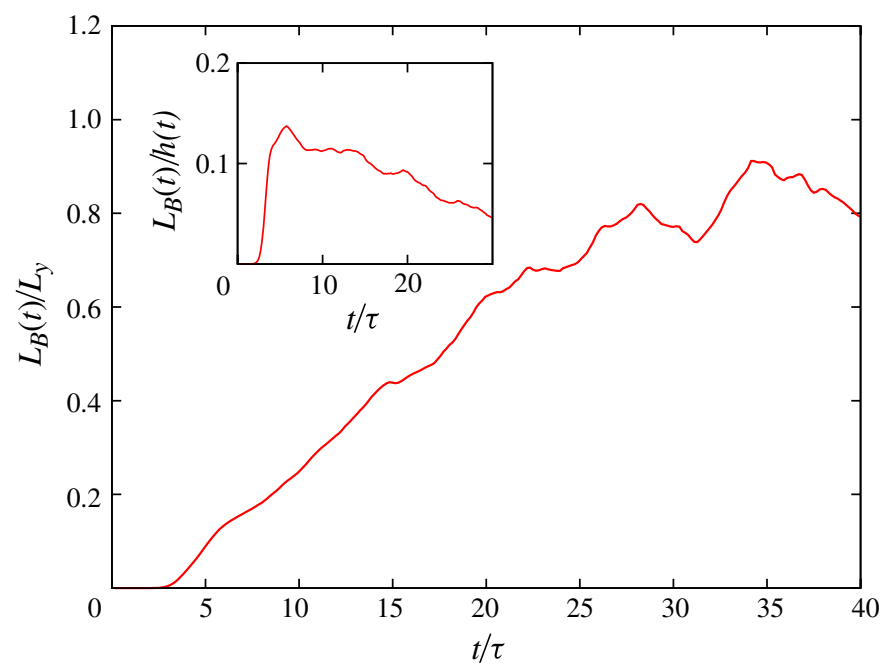

FIGURE 8. (Colour online) Bolgiano scale $L_{B}(2.5)$ normalized with the geometrical scale $L_{y}$ as a function of time. Inset: the same quantity compensated with the mixing layer width $h(t)$.

predictions discussed in $\S 2$. At intermediate wavenumbers, $1 / L_{y}<k<1 / \eta$, we are unable to observe the expected Kolmogorov scaling $k^{-5 / 3}$, probably due to finite Reynolds number effects. Indeed, as discussed above, a much larger scale separation between $\eta$ and $L_{y}$ would be necessary.

The Bolgiano-like spectrum of figure 9 is a strong indication of the presence in the flow of an upscale energy transfer. This can be verified by computing the scaledependent energy flux, given by the third-order structure function of longitudinal velocity increments (i.e. taken along the local velocity direction) $S_{3}(r) \equiv\left\langle\left(\delta_{\|} u(r)\right)^{3}\right\rangle$. We recall that for isotropic three-dimensional turbulence, the classical result due to 


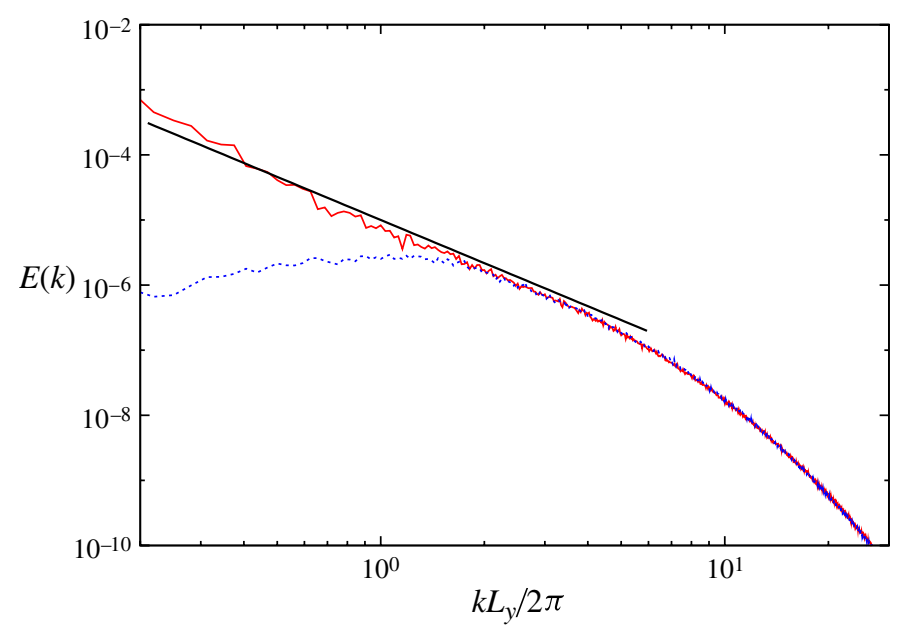

FIgURE 9. (Colour online) Kinetic energy spectra computed at $t=35 \tau$. The thin continuous line represents the components $E_{u}(k)+E_{w}(k)$, the dotted line is $E_{v}(k)$. Spectra are computed by $2 \mathrm{D}$ Fourier transforming the velocity field on $(x, z)$-planes and by averaging over the $y$-direction. The straight line represents Bolgiano scaling $k^{-11 / 5}$.

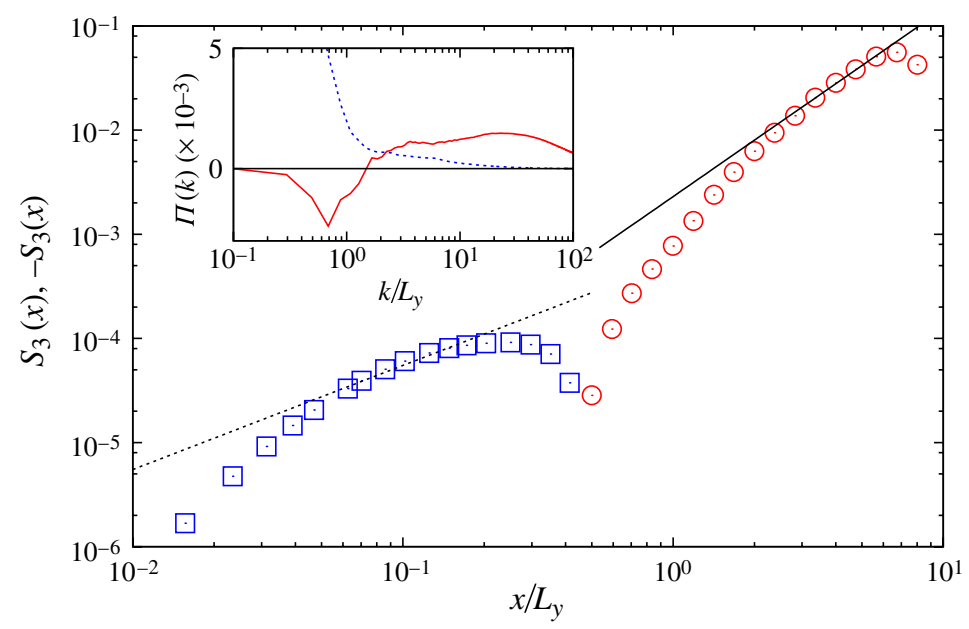

FIGURE 10. (Colour online) Third-order longitudinal velocity structure function $S_{3}(x)$ (circles) and $-S_{3}(x)$ (squares) computed in the central part of the mixing layer along the $x$-direction at time $t=35 \tau$. The two lines represent Kolmogorov scaling $x$ (dotted) and Bolgiano scaling $x^{9 / 5}$ (solid). Inset: contribution to the energy flux in Fourier space $\Pi(k)$ from the nonlinear term (continuous line) and from the buoyancy term (dashed line).

Kolmogorov predicts $S_{3}(r)=-(4 / 5) \varepsilon r$ (Frisch 1995), while positive values of $S_{3}(r)$ indicate the presence of an inverse cascade. Because of the strong inhomogeneity and anisotropy of our flow, we compute structure functions by taking differences of the first component of the velocity in the $r=x$ direction (i.e. the large homogeneous direction), again averaging over the $y$-direction and over the central part of the mixing layer, $|z| \leqslant h / 10$. 


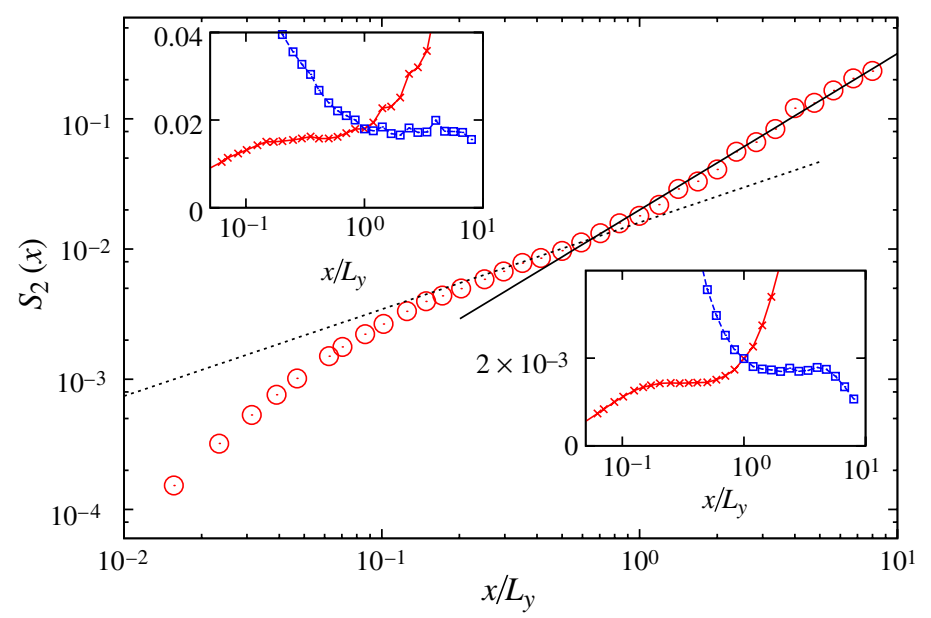

FIGURE 11. (Colour online) Second-order longitudinal velocity structure function $S_{2}(x)$ computed in the central part of the mixing layer along the $x$-direction at time $t=35 \tau$. The two lines represent Kolmogorov scaling $x^{2 / 3}$ (dotted) and Bolgiano scaling $x^{6 / 5}$ (solid). Upper inset: second-order structure function $S_{2}(x)$ compensated with best-fit scaling law at small scales $x^{0.7}$ (crosses) and at large scales $x^{1.3}$ (squares). Lower inset: fourth-order structure function $S_{4}(x)$ compensated with best-fit scaling law at small scales $x^{1.2}$ (crosses) and at large scales $x^{2.45}$ (squares).

As shown in figure 10, at small scales $r<L_{y}, S_{3}(r)$ is negative and, in a narrow range of scales, compatible with the Kolmogorov prediction $S_{3}(r) \sim r$. At scales $r>L_{y}, S_{3}(r)$ becomes positive, signalling the reversal of the energy cascade, and displays a scaling behaviour $S_{3}(r) \sim r^{9 / 5}$ consistent with (2.4). We remark that the absence of clear scaling for the third-order structure function, compared with the spectrum in figure 9, is due to the presence of different inertial ranges (direct and inverse) which have to be simultaneously resolved. Again this is a limitation due to finite-size effects even with the relatively large resolution of our simulations.

The inset of figure 10 shows the contributions of the inertia and buoyancy terms to the energy flux in Fourier space $\Pi(k) \equiv(\mathrm{d} / \mathrm{d} t) \int_{k}^{\infty} E(p) \mathrm{d} p$ where $E(p)$ is the energy spectrum and the time derivative is computed by taking into account, separately, the nonlinear and buoyancy terms of (2.1). At low wavenumbers $k L_{y}<1$, the buoyancy contribution is dominant, and the negative sign of the inertial contribution to the energy flux confirms the presence of a $2 \mathrm{D}$ inverse cascade. At high wavenumbers $k L_{y}>1$ the buoyancy contribution becomes sub-dominant, and one recovers a constant positive flux characteristic of the $3 \mathrm{D}$ regime.

The coexistence of Kolmogorov-Obukhov scaling at small scales and Bolgiano-Obukhov scaling at large scales is confirmed by the behaviour of the structure functions of longitudinal velocity increments $S_{p}(r) \equiv\left\langle\left(\delta_{\|} u(r)\right)^{p}\right\rangle$ for $p=2,4$ shown in figure 11. Because $S_{2}(r)$ does not change sign, the scaling here is more clear than in figure 10 and comparable to the one observed for the spectrum. Two different behaviours are clearly observed at scales smaller and larger than $L_{y}$, respectively compatible with Kolmogorov and Bolgiano scaling (show as lines) and with the interpretation of $L_{y}$ as the Bolgiano scale of the system. Small deviations with respect to the dimensional scaling are observed and can be measured by compensating structure functions with power-law behaviour, $S_{2}(r) \sim r^{\zeta_{2}}$. The result is presented in the insets. Numerically obtained scaling exponents are $\zeta_{2}=0.7 \pm 0.1$ 


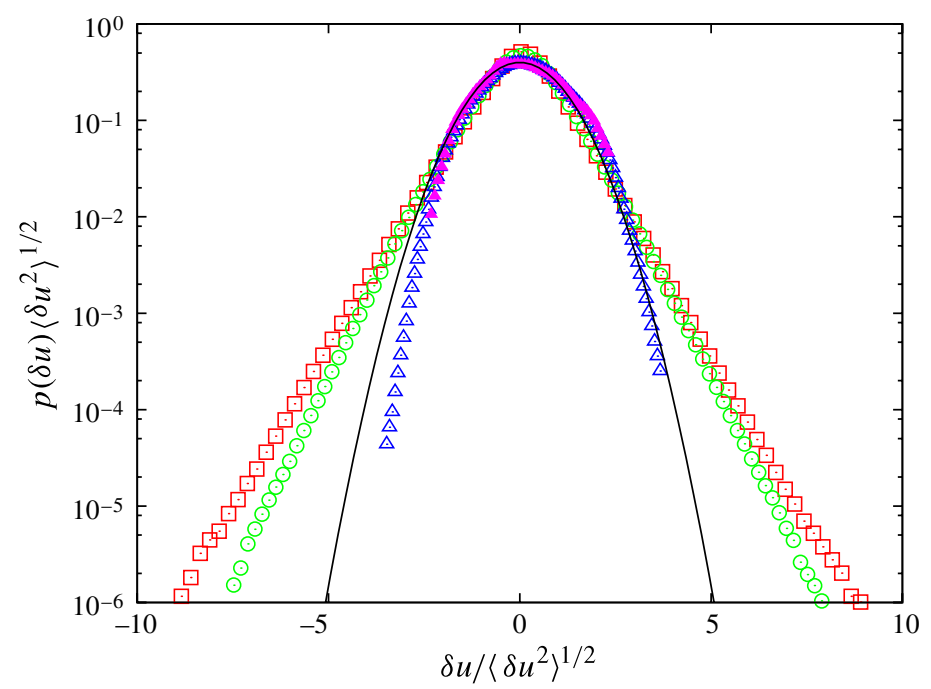

FIgURE 12. (Colour online) Probability density function of longitudinal velocity increments $\delta_{\|} u(r)$ of the $x$-component of the velocity in the $x$-direction at time $t=35 \tau$. The four curves correspond to increments $r=L_{y} / 8$ (squares), $r=L_{y} / 4$ (circles), $r=2 L_{y}$ (open triangles) and $r=4 L_{y}$ (closed triangles). The line represent a Gaussian distribution.

and $\zeta_{2}=1.3 \pm 0.2$ at small and large scales respectively, while for the fourth-order structure function we obtain $\zeta_{4}=1.2 \pm 0.1$ and $\zeta_{4}=2.5 \pm 0.2$. Error estimation is based on the fluctuations of the exponents by changing the range of scales on which compensation is observed. Large-scale exponents are compatible with Bolgiano scaling $\zeta_{p}=3 p / 5$. Small-scale exponents are marginally compatible with Kolmogorov scaling $\zeta_{p}=p / 3$. The observed deviations are in the direction of intermittency corrections in standard three-dimensional turbulence (Frisch 1995).

The presence of small-scale intermittency is confirmed by the shape of the probability density functions of longitudinal velocity increments $\delta_{\|} u(r)$ shown in figure 12. At small scales $\left(r<L_{y}\right)$ they are not self-similar (i.e. they do not rescale with variance) and develop quasi-exponential tails. At large scales $\left(r>L_{y}\right)$ they recover an almost Gaussian form, independent of the scale. The recovery of a Gaussian distribution is consistent with what is observed for inverse cascades in two-dimensional turbulence (Boffetta \& Ecke 2012).

Figure 13 shows the second-order structure function of temperature increments $S_{2}^{T}(r) \equiv\left\langle(\delta T(r))^{2}\right\rangle$, again computed in the central part of the mixing layer along the $x$-axis. Also in this case, a change in the behaviour around $r=L_{y}$ is observable. For $r<L_{y}$ a small range of scaling compatible with Kolmogorov scaling $r^{2 / 3}$ is observed, and at large $r>L_{y}$ a less steep behaviour is found, again compatible with Bolgiano scaling $r^{2 / 5}$. Temperature fluctuation structure functions display much stronger fluctuations than the corresponding velocity structure functions of figure 11, in particular at large scales. This does not allow a precise determination of scaling exponents, as is evident from the compensation with dimensional scaling laws shown in the inset. These fluctuations are probably due to the presence of large-scale regions of unmixed fluid within the mixing layer, as shown by the black and white plumes in figure 1. These structures are clearly $y$-independent and therefore their contribution persists even after averaging over the $y$-direction. 


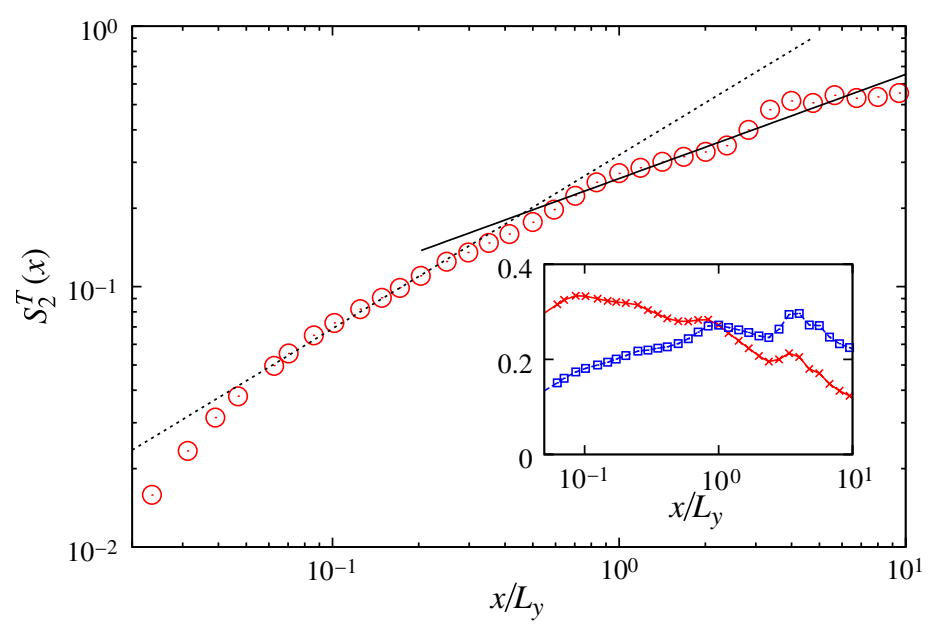

FIgURE 13. (Colour online) Second-order temperature structure function $S_{2}^{T}(x)$ computed in the central part of the mixing layer along the $x$-direction at time $t=35 \tau$. The two lines represent Kolmogorov scaling $r^{2 / 3}$ (dotted) and Bolgiano scaling $r^{2 / 5}$ (solid). Inset: secondorder structure function $S_{2}^{T}(r)$ compensated by Kolmogorov (crosses) and Bolgiano (squares) scaling.

\section{Conclusions}

We have investigated, by means of stat-of-the-art numerical simulations of primitive equations, the scaling properties of temperature and velocity fluctuations in Rayleigh-Taylor turbulent convection confined in a box with small aspect ratio. We have shown that the system undergoes a dimensional transition from three- to two-dimensional dynamics, which occurs when the width of the mixing layer becomes larger than the confining scale $L_{y}$, which becomes the Bolgiano scale of the system. In the late stage of the evolution we observed the coexistence of two different scaling regimes. At scales larger than $L_{y}$ the two-dimensionalization of the flow induced by the confinement gives rise to an inverse energy cascade which is associated with the appearance of Bolgiano-Obukhov scaling. However, a remnant flux of energy toward small scales originates a three-dimensional turbulent motion at scales smaller than $L_{y}$ which is characterized by Kolmogorov-Obukhov scaling. This result is consistent with recent findings obtained within the framework of shell models for confined convective turbulence (Boffetta, De Lillo \& Musacchio 2011).

It is interesting to note that in the case studied here, the presence of Bolgiano-Obukhov scaling is strictly connected to the presence of an energy transfer toward large scales. An upscale energy transfer in three-dimensional flows has been obtained not only in the case of geometrical confinement, but also in the case of rotating turbulence (see e.g. Métais et al. 1996; Smith \& Waleffe 1999, 2002; Chen et al. 2005; Waite \& Bartello 2006; Mininni, Alexakis \& Pouquet 2009). In particular Chen et al. (2005) observed, at small Rossby number, an inverse energy cascade for the slow modes of the velocity field, with the characteristics of two-dimensional turbulence. This poses the intriguing question of whether in other turbulent convective setups the Bolgiano-Obukhov phenomenology could be observed whenever an upscale energy transfer is induced in the turbulent flow by geometrical constraints and/or physical mechanisms (such as rotation or stratification). 
We mention that recent experiments on soap film convection observed Bolgiano scaling for large values of the Rayleigh number (Zhang \& Wu 2005; Seychelles et al. 2010). Despite the similarities with our results, we note the presence of important differences, as the experimental data show both the presence of intermittency in the velocity field (Zhang \& Wu 2005) and the absence of intermittency in the temperature field (Seychelles et al. 2010). Moreover, the direction of the energy flux was not reported. Our simulations indicate the absence of intermittency for the velocity field, which exhibits an inverse cascade, and are compatible with some intermittency in the direct cascade. It would therefore be extremely interesting to have a direct comparison of experimental and numerical data on thermal convection in quasi-two-dimensional flow which would give new insights into the fundamental issues of scaling in turbulent convection.

\section{Acknowledgements}

Numerical simulations have been done at the Cineca under the HPC-EUROPA2 project (project number: 228398) with the support of the European Commission - Capacities Area - Research Infrastructures. We thank G. Erbacci and the staff at Cineca Supercomputing Center (Bologna, Italy) for their support.

\section{REFERENCES}

Ahlers, G., Grossmann, S. \& Lohse, D. 2009 Heat transfer and large scale dynamics in turbulent Rayleigh-Bénard convection. Rev. Mod. Phys. 81 (2), 503-537.

Biferale, L., Mantovani, F., Sbragaglia, M., Scagliarini, A., Toschi, F. \& Tripiccione, R. 2010 High resolution numerical study of Rayleigh-Taylor turbulence using a thermal lattice Boltzmann scheme. Phys. Fluids 22, 115112.

Boffetta, G., De Lillo, F. \& Musacchio, S. $2010 a$ Nonlinear diffusion model for Rayleigh-Taylor mixing. Phys. Rev. Lett. 104, 034505.

Boffetta, G., De Lillo, F. \& Musacchio, S. 2011 Shell model for quasi-two-dimensional turbulence. Phys. Rev. E 83 (6), 066302.

Boffetta, G. \& Ecke, R. E. 2012 Two-dimensional turbulence. Annu. Rev. Fluid Mech. 44, 427.

Boffetta, G., Mazzino, A., Musacchio, S. \& Vozella, L. 2009 Kolmogorov scaling and intermittency in Rayleigh-Taylor turbulence. Phys. Rev. E 79 (6), 065301(R).

Boffetta, G., Mazzino, A., Musacchio, S. \& Vozella, L. $2010 b$ Statistics of mixing in three-dimensional Rayleigh-Taylor turbulence at low Atwood number and Prandtl number one. Phys. Fluids 22, 035109.

Bolgiano, R. 1959 Turbulent spectra in a stably stratified atmosphere. J. Geophys. Res. 64 (12), 2226-2229.

Савот, W. 2006 Comparison of two- and three-dimensional simulations of miscible Rayleigh-Taylor instability. Phys. Fluids 18 (4), 045101.

Савот, W. H. \& CoOK, A. W. 2006 Reynolds number effects on Rayleigh-Taylor instability with possible implications for type Ia supernovae. Nature Phys. 2 (8), 562.

Celani, A., Mazzino, A. \& Vozella, L. 2006 Rayleigh-Taylor turbulence in two dimensions. Phys. Rev. Lett. 96 (13), 134504.

Celani, A., Musacchio, S. \& Vincenzi, D. 2010 Turbulence in more than two and less than three dimensions. Phys. Rev. Lett. 104 (18), 184506.

Chen, Q., Chen, S., Eyink, G. \& Holm, D. D. 2005 Resonant interactions in rotating homogeneous three-dimensional turbulence. J. Fluid Mech. 542, 139-164.

Chertkov, M. 2003 Phenomenology of Rayleigh-Taylor turbulence. Phys. Rev. Lett. 91 (11), 115001.

CoRrsin, S. 1951 On the spectrum of isotropic temperature fluctuations in an isotropic turbulence. J. Appl. Phys. 22 (4), 469-473. 
Dalziel, S. B., Linden, P. F. \& Youngs, D. L. 1999 Self-similarity and internal structure of turbulence induced by Rayleigh-Taylor instability. J. Fluid Mech. 399, 1-48.

Frisch, U. 1995 Turbulence: The Legacy of AN Kolmogorov. Cambridge University Press.

Isobe, H., Miyagoshi, T., Shibata, K. \& Yokoyama, T. 2005 Filamentary structure on the Sun from the magnetic Rayleigh-Taylor instability. Nature 434 (7032), 478-481.

Kraichnan, R H \& Montgomery, D 1980 Two-dimensional turbulence. Rep. Prog. Phys. 43 (5), 547-619.

Lohse, D. \& XIA, K. Q. 2010 Small-scale properties of turbulent Rayleigh-Bénard convection. Annu. Rev. Fluid Mech. 42, 335-364.

Matsumoto, T. 2009 Anomalous scaling of three-dimensional Rayleigh-Taylor turbulence. Phys. Rev. E 79 (5), 055301(R).

Métais, O., Bartello, P., Garnier, E., Riley, J. J. \& Lesieur, M. 1996 Inverse cascade in stably-stratified rotating turbulence. Dyn. Atmos. Oceans 23, 193-203.

Mininni, P. D., Alexakis, A. \& Pouquet, A. 2009 Scale interactions and scaling laws in rotating flows at moderate rossby numbers and large Reynolds numbers. Phys. Fluids 21, 015108 .

Ngan, K., Straub, D. N. \& Bartello, P. 2005 Aspect ratio effects in quasi-two-dimensional turbulence. Phys. Fluids 17, 125102.

Oвukhov, A. M. 1949 Structure of the temperature field in a turbulent current. Izv. Akad. Nauk SSSR Geogr. Geofiz. 13 (1), 58-69.

OвukHov, A. 1959 Effect of Archimedean forces on the structure of the temperature field in a turbulent flow. Dokl. Akad. Nauk SSSR 125, 1246.

RistorCELLI, J. R. \& CLARK, T. T. 2004 Rayleigh-Taylor turbulence: self-similar analysis and direct numerical simulations. J. Fluid Mech. 507, 213-253.

Schultz, D. M., KAnAK, K. M. \& Straka, J. M. 2006 The mysteries of mammatus clouds: observations and formation mechanisms. J. Atmos. Sci. 63, 2409-2435.

Seychelles, F., Ingremeau, F., Pradere, C. \& Kellay, H. 2010 From intermittent to nonintermittent behaviour in two dimensional thermal convection in a soap bubble. Phys. Rev. Lett. 105 (26), 264502.

Shats, M., BYRne, D. \& XiA, H. 2010 Turbulence decay rate as a measure of flow dimensionality. Phys. Rev. Lett. 105 (26), 264501.

Shraiman, B. I. \& Siggia, E. D. 1990 Heat transport in high-Rayleigh-number convection. Phys. Rev. A 42 (6), 3650-3653.

Siggia, E. D. 1994 High Rayleigh number convection. Annu. Rev. Fluid Mech. 26, 137-168.

Smith, L. M., Chasnov, J. R. \& WalefFe, F. 1996 Crossover from two- to three-dimensional turbulence. Phys. Rev. Lett. 77 (12), 2467-2470.

SMith, L. M. \& WALEFFe, F. 1999 Transfer of energy to two-dimensional large scales in forced, rotating three-dimensional turbulence. Phys. Fluids 11 (6), 1608-1621.

SMith, L. M. \& WALEFFE, F. 2002 Generation of slow large scales in forced rotating stratified turbulence. J. Fluid Mech. 451, 145-168.

Vladimirova, N. \& ChertKov, M. 2009 Self-similarity and universality in Rayleigh-Taylor, Boussinesq turbulence. Phys. Fluids 21, 015102.

Waite, M. L. \& BARTEllo, P. 2006 The transition from geostrophic to stratified turbulence. J. Fluid Mech. 568, 89-108.

Xia, H., Byrne, D., Falkovich, G. \& Shats, M. 2011 Upscale energy transfer in thick turbulent fluid layers. Nature Phys. 7, 321-324.

ZHANG, JIE \& WU, X. L. 2005 Velocity intermittency in a buoyancy subrange in a two-dimensional soap film convection experiment. Phys. Rev. Lett. 94 (23), 234501. 